\title{
Performance Impacts of Autocorrelated Flows in Multi-tiered Systems
}

\author{
Ningfang Mi \\ College of William and Mary \\ Williamsburg, VA, USA \\ ningfang@cs.wm.edu
}

\section{INTRODUCTION}

We focus on the general problem of capacity planning and performance prediction of multi-tiered systems. Workload characterization studies of such systems usually examine the stochastic characteristics of arrivals to the system and wait/service times at various tiers aiming at bottleneck identification, diagnosing the conditions under which bottlenecks are triggered, and assisting the development of resource management policies to improve performance or provide service level provisioning.

In this paper, we examine how burstiness in the arrival or service process of any of the tiers affects end-to-end performance. More specifically, our focus is on systems where there is always an $u p$ per bound on the number of jobs that are in the system at all times. A system with finite buffers and/or admission control, which is indeed the case in a multi-tiered system as buffer sizes or the maximum number of simultaneous connections is restricted by software and/or hardware, behaves in essence like a closed system, i.e., a system with a closed loop structure [3].

In multi-tiered systems with a closed loop structure, if autocorrelation exists in the service process of any of the tiers, then it propagates across the entire loop in the closed system and is present in the arrival stream of tiers (queues) that precede that tier (queue), unexpectedly affecting their performance as well as end-to-end performance. Comparing the performance effects of the presence of autocorrelated flows in multi-tiered systems with the performance of the same system with independent flows, we show that end-toend performance significantly deteriorates while bottleneck devices are less utilized, falsely indicating that the system is able to sustain higher load. To the best of our knowledge, this is the first time that autocorrelated flows are identified as an important stochastic characteristic in multi-tiered systems with a closed-loop structure.

\section{AUTOCORRELATION IN CLOSED SYS- TEMS}

We use the simplest closed queuing system that resembles the topology of a multi-tiered application. The purpose of this analysis is twofold: (1) to observe how autocorrelation propagates through all tiers in the system and (2) to observe how autocorrelation affects system performance. Considering a closed queuing network with two queues only, we let the service time of $Q_{1}$ be autocorrelated and drawn from a 2-state Markovian-Modulated Poisson Process (MMPP) with mean $1 / \mu_{1}=0.5, S C V=20$, and let the service process of $Q_{2}$ be exponentially distributed with mean service time $1 / \mu_{2}=1$.

Figure 1 illustrates the autocorrelation propagation for population $N=50$. The ACF of the MMPP(2) that generates the service times at $Q_{1}$ is also shown in the figure. We can see that autocorrelation propagates through the tiers, but with reduced strength com-

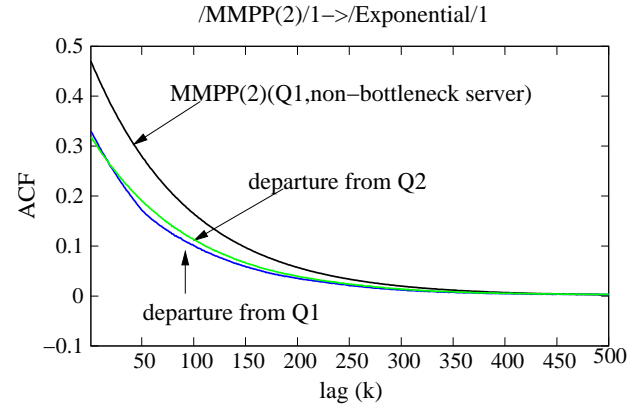

Figure 1: The ACF of departures from $Q_{1}$ (arrivals to $Q_{2}$ ), departures from $Q_{2}$ (arrivals to $Q_{1}$ ). The ACF of the service process that generates autocorrelated flows in the system is also illustrated.

pared to the autocorrelation of the service process that injected autocorrelation into the system (i.e., $Q_{1}$ ). Any autocorrelation in the flows into $Q_{2}$ is reduced as departures from this queue are spaced further apart thanks to the slower exponential service times of $Q_{2}$.

We now turn to the effects of autocorrelation on system performance. Autocorrelated flows in the closed system degrade overall system performance, i.e., increase mean round trip time and decrease mean throughput, see Figure 2. They also decrease the expected utilization of each queue, including the expected utilization at the bottleneck device. This is because that autocorrelation in both arrival and service processes of the non-bottleneck $Q_{1}$ queue degrades performance. More customers accumulate there and cause mean queue length of the bottleneck to decrease, as requests spend now more time in $Q_{1}$. This redistribution of requests in the system "balances" the load, as it is also reflected in the per queue utilizations. For the experiments with ACF, we see that throughput increases very slowly as $N$ increases, consistent with the very slow growth of utilization at the bottleneck queue. Indeed, maximum throughput and the corresponding $99.5 \%$ utilization are reached with much higher $N=3000$ for the ACF experiments. Therefore, throughput and utilization, metrics that are easily obtainable from measurements and are prevalently used to gauge system capacity, give a distorted view of the user-perceived performance.

\section{EXPERIMENTAL CASE STUDY: TPC-W}

In this section, we present a case study based on the TPC-W benchmark, a widely used e-commerce benchmark to simulate the behavior of a Business-to-Consumer (B2C) site [1], and devise a model to capture the observed behavior.

We observe that autocorrelation propagates across all the tiers in 
(a) Average response time

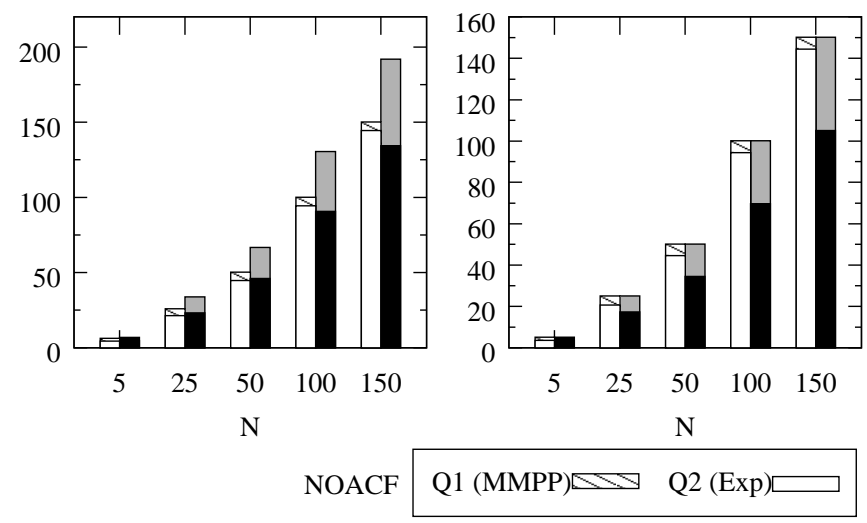

(c) Average utilization

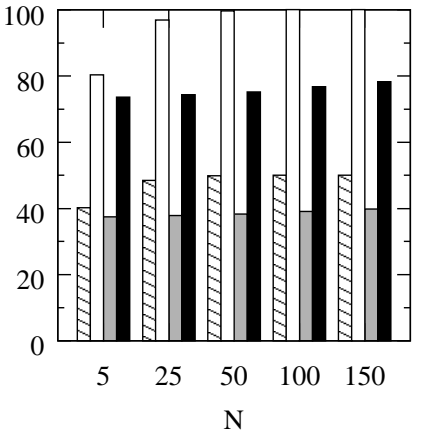

$\mathrm{ACF} \quad \mathrm{Q} 2(\mathrm{MMPP}) \square \mathrm{Q} 1(\operatorname{Exp}) \square$ (d) Average throughput

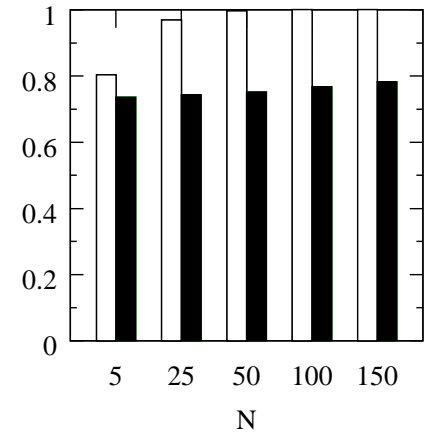

$\mathrm{N}$

Figure 2: Performance measures: (a) mean round trip time, (b) mean queue length, (c) mean utilization, and (d) mean throughput at each queue. NOACF indicates that $Q_{1}$ has independent service times, ACF indicates that $Q_{1}$ has autocorrelated service times.

(a) Average response times

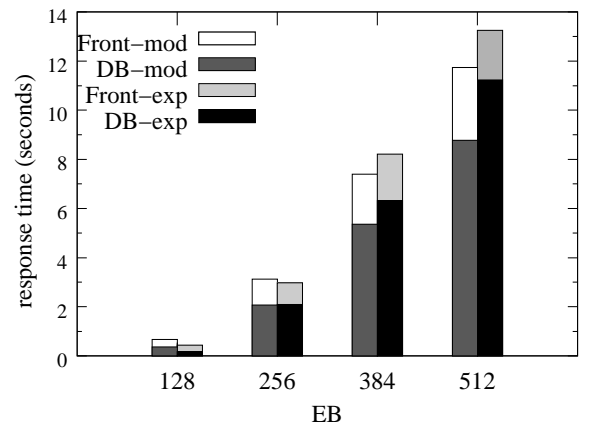

(b) Average queue lengths

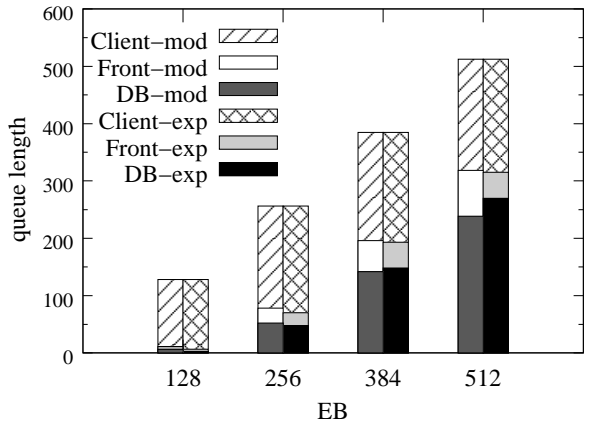

(c) Average utilization

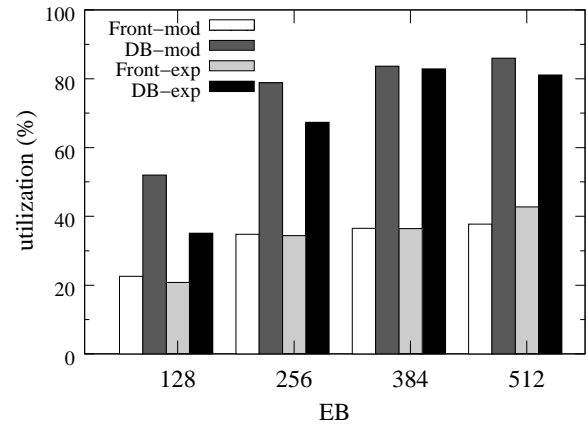

Figure 3: Model prediction and experimental performance measures.

a multi-tiered system and we attribute its presence to autocorrelated service processes in the front and/or database servers because the workload generation at the customers or emulated browsers (EBs) guarantees that there is no autocorrelation in the arrival process coming from the clients.

Average performance values are presented in Figure 3. Despite the fact that queue lengths and response times increase fast as a function of EBs (see Figures 3(a) and (b)), utilization levels increase very slowly, consistently with the results presented in Section 2. The performance measures from the model are also presented in the figure. Model and experimental results are in excellent agreement, in spite of the fact that no rigorous fitting method was used to fit the experimentally collected service times and their autocorrelation of the front-end server.

\section{SUMMARY}

We presented a performance evaluation study that shows the presence of autocorrelated flows in a multi-tiered system with a closedloop structure and their performance effects. Via queuing models we have shown that identifying autocorrelated flows in such a system is critical for capacity planning. If autocorrelated flows are ignored, then throughput and utilization of specific devices - metrics often used in capacity planning and admission control - may give a distorted view of system load. For more details, we direct the reader to [2].

Future work on the theoretical side will be to develop analytic models of closed systems that consider autocorrelation. Such mod- els could be used to identify the conditions in a closed system that trigger autocorrelation, as well as the conditions that augment or reduce autocorrelation. On the systems side, we are working on new resource management techniques that take autocorrelation into account. We also intend to widen the set of workloads considered and understand in more detail how autocorrelation arises in the service process.

\section{REFERENCES}

[1] D. Garcia and J. Garcia. TPC-W E-commerce benchmark evaluation. IEEE Computer, pages 42-48, Feb. 2003.

[2] N. Mi, Q. Zhang, A. Riska, and E. Smirni. Performance Impacts of Autocorrelated Flows in Multi-tiered Systems. In Proceedings of the 26th International Symposium on Computer Performance, Modeling, Measurements, and Evaluation (Performance 2007), Cologne, Germany, Oct. 2007.

[3] R. O. Onvural and H. G. Perros. Equivalencies between open and closed queueing networks with finite buffers. Performance Evaluation, 9:263-269, 1989. 